Laser Chem. 1988, Vol. 9, pp. 219-240

(C) 1988 Harwood Academic Publishers GmbH

Photocopying permitted by license only

Reprints available directly from the Publisher

Printed in the United Kingdom

\title{
Velocity Dependence of Rotationally Inelastic Cross-Sections
}

\author{
CHRISTOPHER P. FELL, ANTHONY J. MCCAFFERY, KATHARINE L. \\ REID, ANTON TICKTIN and BENJAMIN J. WHITAKER
}

School of Molecular Sciences, University of Sussex, Brighton BN1 9OJ, UK

(Received 20 May, 1988)

Techniques are described for obtaining the energy-dependence of state to state rotational energy transfer (RET) cross-sections in thermal cell experiments through velocity selection by Doppler shift. The case of polarised excitation and detection is discussed in detail and expressions presented which enable the state multipoles of population, orientation and alignment to be obtained from appropriate RET experiments. It is found that the translational order present in a non-isotropic collision environment, such as that resulting from excitation with a narrow linewidth laser, causes state multipoles of different rank to become coupled. Experimental results are presented for the energy dependence of cross-sections for state to state transfer of population and of orientation in $\mathrm{Li}_{2}-\mathrm{Xe}$ collisions. The way the relative velocity vector changes as a result of a collision may also in principle be studied using a high resolution probe (e.g. a second laser) of the velocity spread following RET.

KEY WORDS: Energy-dependence, rotational energy transfer (RET).

\section{INTRODUCTION}

The study of line profiles of spectroscopic transitions has long been a rich source of information on the dynamics of molecular interactions. Pressure broadening of the homogeneous component of spectral lineshapes for example has yielded valuable data on inter-particle potentials along with relaxation rates. For gas phase systems, the dominant mechanism responsible for broadening a spectral transition 
beyond its natural linewidth is of course the Doppler effect. Recent studies of molecular collision dynamics have made use of this to obtain information on the velocity dependence of dynamical processes. Particularly influential, are those on rotationally inelastic collisions ${ }^{1}$ and on photodissociation. The latter experiments have been reviewed by Houston. $^{2}$

In this contribution we concentrate on rotationally inelastic collisions in atom-diatom systems and make use of the velocity selection available by probing with lasers of linewidths narrower than the Doppler profile of a transition to a specific $j$ level in an excited electronic state. We shall pay particular attention to the extra information that is available from experiments using full polarisation selection and detection and describe an experiment that measures the polarisation of resolved fluorescence following RET.

The material is organised as follows. We first discuss the rate constants for rotationally inelastic processes for a selected molecular velocity component. Second, the multipolar cross-sections appropriate to the cylindrical symmetry that is a consequence of laser excitation are discussed. The methods for deconvolution of experimental data to yield energy dependent cross-sections is briefly described and data are presented for the case of $\mathrm{Li}_{2}-\mathrm{Xe}$ rotationally inelastic collisions. Finally we give a very brief account of a velocityselected entrance channel-Doppler scanned exit channel experiment from which differential cross-sections may in principle be obtained.

\section{VELOCITY SELECTION BY DOPPLER SHIFT}

Rotational energy transfer (RET) experiments using the laser fluorescence technique on molecules in thermal equilibrium (cell experiments) can be carried out under a range of excitation conditions. If a broad band laser source is used (several $\mathrm{GHz}$ for the systems discussed below), then all molecular velocity components will be equally excited and the excited state will consist of a thermal distribution of velocities. The rate constant of RET for such a system is ${ }^{3}$

$$
k_{j \mu \rightarrow j^{\prime} \mu^{\prime}}(T)=C \int_{0}^{\infty} v_{r}^{3} e^{-\beta v_{r}^{2}} \sigma_{j \mu \rightarrow j^{\prime} \mu^{\prime}}\left(v_{r}\right) d v_{r},
$$


where $v_{r}$ is the relative velocity, $C$ a constant, and $\sigma_{j \mu \rightarrow j^{\prime} \mu^{\prime}}\left(v_{r}\right)$ the cross-section as a function of velocity for the transfer from initial state $j \mu$ to final state $j^{\prime} \mu^{\prime}$ with $\mu, \mu^{\prime}$ referred to the laboratory frame (LF) axis. $\beta$ is the reduced mass (for notational convenience the $2 \mathrm{kT}$ factor has been dropped from all velocity integrations). As well as determining cross-sections for RET from such experiments it is possible to obtain information on how the direction of rotational angular momentum changes as a result of collision by measuring the polarisation of the emitted fluorescence. This is possible because laser excitation creates an anisotropic distribution of $\mu$ states depending on the laser polarisation. Although anisotropic this distribution has symmetry properties which may be described using the state multipole formalism. ${ }^{4}$ Single photon excitation may produce only multipoles up to order $K=2$. In an isotropic collision environment each state multipole relaxes independently the RET process being described by the cross-sections $\sigma_{i j^{\prime}}^{K} .5$

If a sufficiently narrow linewidth dye laser is used then specific components of molecular velocity will be selected. For a given excitation frequency, the relationship between that frequency and the velocity selected is given by

$$
\omega=\omega_{0}+k v_{m z},
$$

where the laser beam propagation direction is taken to be the $z$-axis and $v_{m z}$ is the component of the velocity of the diatom along this axis, i.e. that selected by the laser. The molecular system has only two degrees of translational freedom, $v_{m x}, v_{m y}$ for this type of experiment, as $v_{m z}$ is determined in the process of tuning the laser frequency. The probability of finding a molecular speed, $v_{m}$, for a given selected $v_{m z}$ is given by

$$
W\left(v_{m}, v_{m z}\right)=\sqrt{v_{m}^{2}-v_{m z}^{2}} e^{-M_{m} v_{m}^{2}},
$$

where $M_{m}$ is the molecular mass. Figure 1 displays a 3-D plot of $W\left(v_{m}\right.$, $v_{m z}$ ) for the case of $\mathrm{Li}_{2}$ at $1000 \mathrm{~K}$ but this serves only to illustrate the spread of the distribution not the relative magnitudes for each $v_{m z}$. It is apparent from this that a tunable single mode dye laser can be used as a molecular speed probe simply by scanning over the Doppler profile. It should be noted that although a very narrow distribution of molecular 


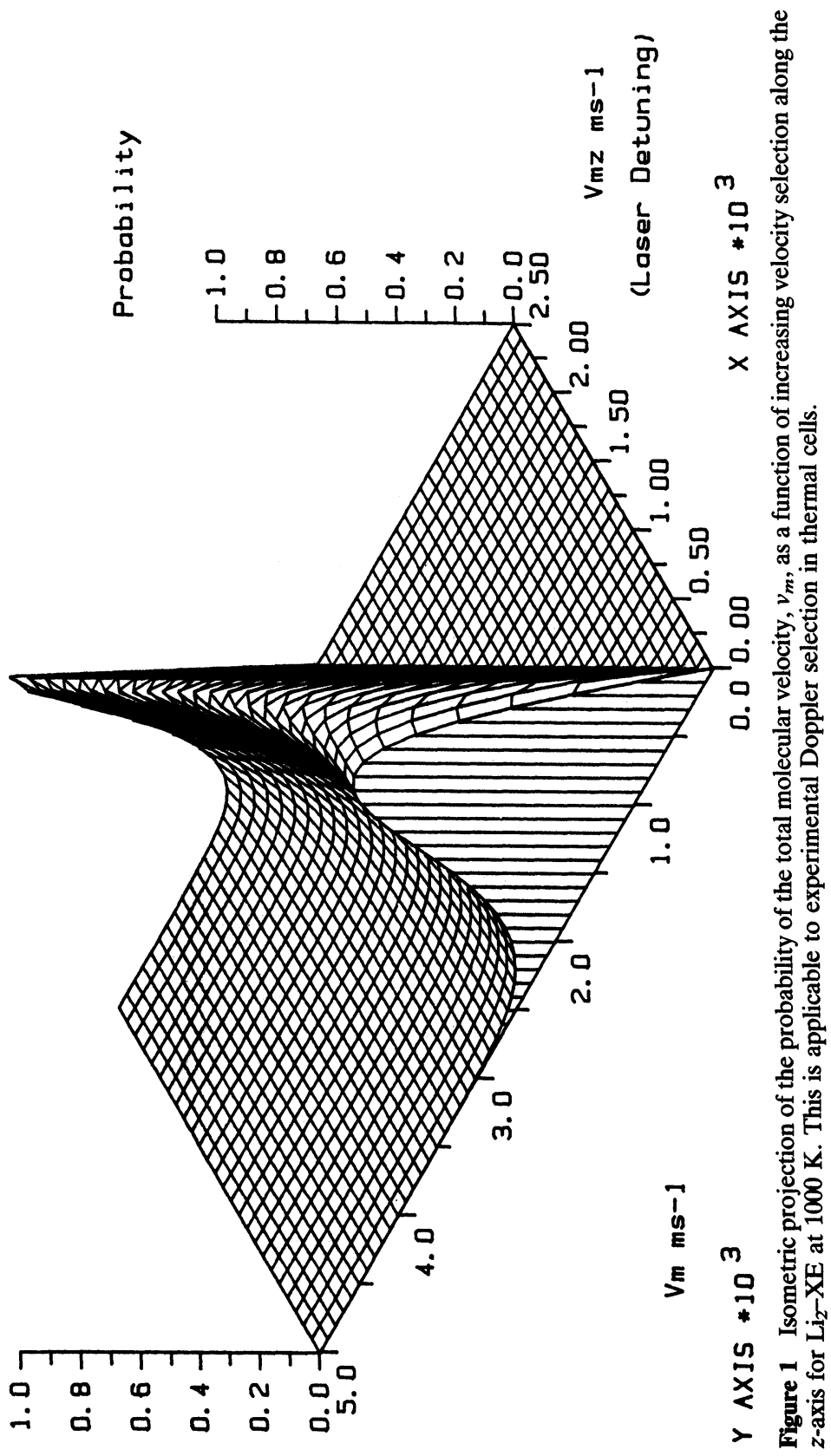


speeds can be selected by tuning the laser to the wings of the Doppler profile, in practice it is difficult to make use of this because the unnormalised probabilities become so small in magnitude.

There is also information on the direction of the molecular velocity for each excitation frequency. For example, when $v_{m z}=0$ (line centre) the angle, $\theta$, between $v_{m}$ and the $z$ axis must be $90^{\circ}$ and so there is a cylindrically symmetric velocity distribution $\left(v_{m x}\right.$ and $v_{m y}$ retain their thermal distribution). When the laser frequency is not at line centre the angular distribution of the molecular velocities is given by:

$$
W\left(\theta, v_{m z}\right)=v_{m z} \tan \theta \exp \left(-M_{m} v_{m z} \sec ^{2} \theta\right),
$$

where we have used the fact that $v_{m z}=v_{m} \cos \theta$. If $v_{m z} \neq 0$, then because of the thermal distribution of the other two molecular velocity components, $\theta$ will have a range of values determined by Eq. (4). Figure 2 displays the angular distributions as a function of $v_{m z}$ for $\mathrm{Li}_{2}$ at $1000 \mathrm{~K}$. As can clearly be seen the most probable value of $\theta$ decreases as $v_{m z}$ increases, and the FWHM spread in $\theta$ varies considerably with $v_{m z}$. At line centre and in the far wings, the range of possible values of $\theta$ is small and there is the possibility of close correlation in these regions between the relative velocity vector and the rotational angular momentum vector if polarised light is used in the excitation process.

It should be clear from the foregoing discussion that a wide range of molecular velocities may be sampled by Doppler selection and that there is asymmetry in the distribution of the velocity vectors. In a thermal cell in which a laser-selected molecule undergoes collisions the rate constant for RET looks more complicated than for a molecule undergoing collisions in an isotropic manner because the usual thermal averages cannot be carried out. There are five unselected Maxwellian velocity components, two for the laser-selected molecule and three for the atom. The rate constant expressions Eq. (1) can be rewritten for such a process explicitly as:

$$
\begin{aligned}
k_{j \mu \rightarrow j^{\prime} \mu^{\prime}}\left(v_{m z}\right)= & C \int_{-\infty}^{\infty} e^{-M_{m}\left(v_{m x}^{2}+v_{m y}^{2}\right)} \sigma_{j \mu \rightarrow j^{\prime} \mu^{\prime}}\left(\bar{v}_{m}-\bar{v}_{a}\right)\left|\bar{v}_{m}-\bar{v}_{a}\right| \\
& \times e^{-M_{a}\left|v_{a}\right|_{2}} d v_{m x} d v_{m y} d v_{a x} d v_{a y} d v_{a z},
\end{aligned}
$$

in which the subscripts $a$ and $m$ refer to the atomic and molecular velocities respectively. To get this equation in terms of the relative 


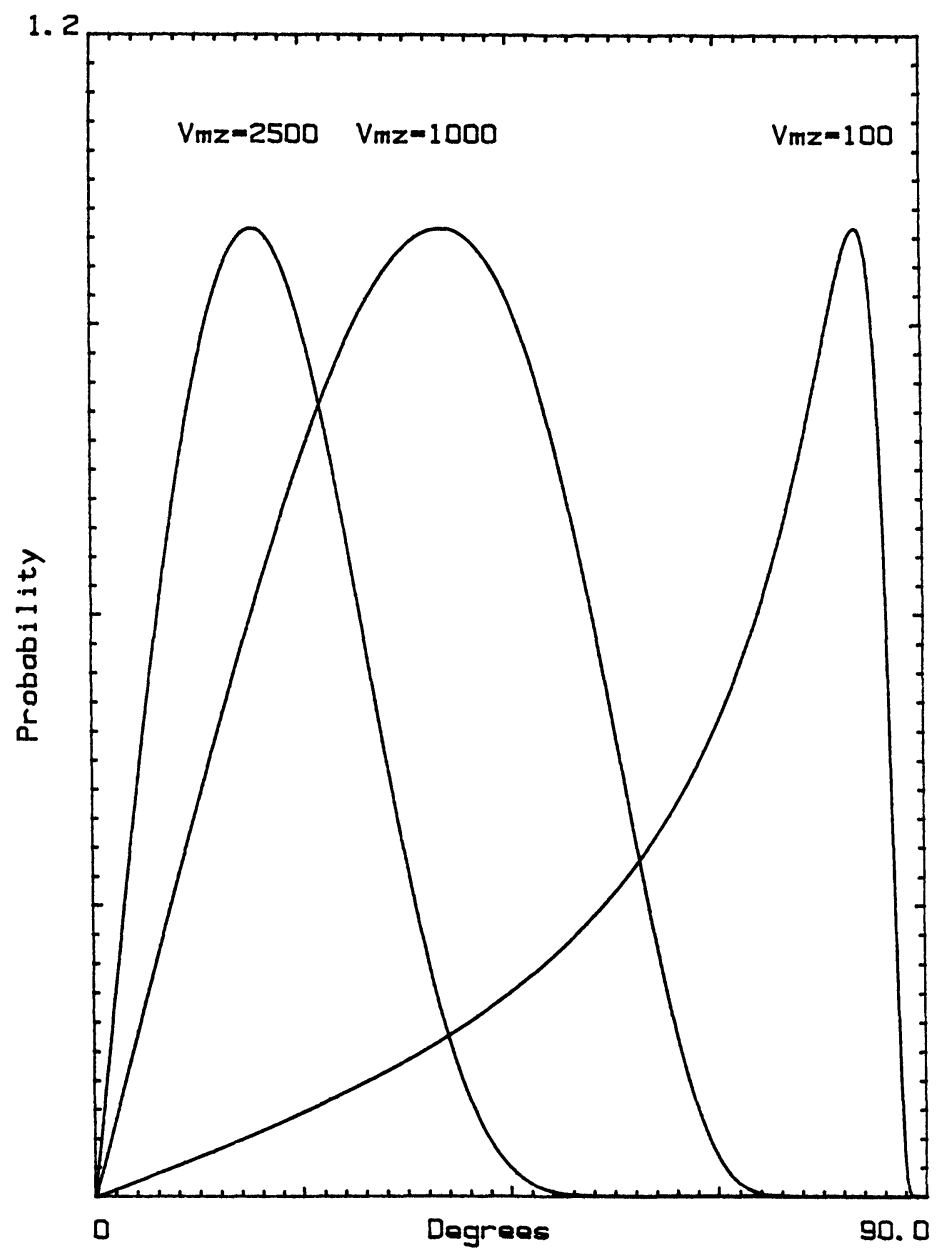

Figure 2 A plot, for $\mathrm{Li}_{2}$ at $1000 \mathrm{~K}$, of the angle of the molecular velocity with respect to the laser beam for three values of $v_{m z}$. The angular spread is small only in the wings and at line centre. (The results have been normalised).

speed, $v_{r}$, we use the relation:

$$
v_{r x}=v_{m x}-v_{a x} \text { for } x \text { and } y
$$

and integrate over $v_{m x}$ and $v_{m y}$ using the identity

$$
\int_{-\infty}^{\infty} e^{-a t^{2}+2 b t+c} d t=1 / 2(\pi / a)^{1 / 2} e^{\left(b^{2}-a c\right) / a},
$$


to give:

$$
\begin{aligned}
k_{j \mu \rightarrow j^{\prime} \mu^{\prime}}\left(v_{m z}\right)= & C \int_{-\infty}^{\infty} e^{-\beta\left(v_{r x}^{2}+v_{r y}^{2}\right)} e^{-m_{a}\left(v_{r x}^{2}+v_{r y}^{2}\right)} v_{r} \\
& \times \sigma_{j \mu \rightarrow j^{\prime} \mu^{\prime}}\left(\bar{v}_{r}\right) d v_{r x} d v_{r y} d v_{r z} .
\end{aligned}
$$

Expressing the relative velocity in polar co-ordinates $v_{r}, \alpha$ and $\phi$, where $\alpha$ and $\phi$ denote the orientation of the laser axis with respect to the initial relative velocity vector, leads to:

$$
\begin{aligned}
k_{j u \rightarrow j^{\prime} \mu^{\prime}}\left(v_{m z}\right)= & C \int_{0}^{2 \pi} \int_{-1}^{1} \int_{0}^{\infty} e^{-\beta\left(v_{r}^{2} \sin ^{2} \alpha\right)} e^{-m_{a}\left(v_{m z}-v_{r} \cos \alpha\right)^{2}} v_{r}^{3} \\
& \times \sigma_{j \mu \rightarrow j^{\prime} \mu^{\prime}}\left(\bar{v}_{r}\right) d v_{r} d(\cos \alpha)^{\prime} d \phi .
\end{aligned}
$$

At first sight it may seen strange that a cross-section can be dependent on angles related to a lab fixed axis. This arises because of the anisotropic distribution of relative velocity vectors created by the laser excitation; a distribution that varies with selected velocity. This in turn means that the relationship between $j$ and $v_{r}$ depends on the selected velocity so that $\alpha$ affects two collision frame vectors.

The key to obtaining energy dependent cross-sections is contained in Eq. (9). It shows that the probability of finding a relative speed $v_{r}$ for a given excited $v_{m z}$ is far from Maxwell-Boltzmann. Hence, by selecting a molecular velocity component we are sampling a given range of collision energies that may be very narrow if we choose collision partners such that the atomic mass is much greater than the molecular mass, and if we excite in the wings of the Doppler profile.

The LF cross-sections can be expressed in terms of collision frame quantities: $^{6}$

$$
\begin{aligned}
\sigma_{j \mu \rightarrow j^{\prime} \mu^{\prime}}\left(v_{r}, \alpha, \phi\right)= & \sum_{m, m_{1}, m^{\prime}, m_{1}^{\prime}} D_{\mu m}^{j}(\alpha, \phi, 0) D_{\mu m_{1}}^{j^{*}}(\alpha, \phi, 0) \\
& \times D_{\mu^{\prime} m^{\prime}}^{j^{\prime}}(\alpha, \phi, 0) D_{\mu m_{1}^{\prime}{ }_{1}}^{j^{*}}(\alpha, \phi, 0) \sigma_{j m m_{1} \rightarrow j^{\prime} m^{\prime} m_{1}^{\prime}}\left(v_{r}\right),
\end{aligned}
$$

where

$$
\sigma_{j m m_{1} \rightarrow j^{\prime} m^{\prime} m_{1}^{\prime}}\left(v_{r}\right) \propto \int f_{j_{1} m \rightarrow j^{\prime} m_{1}^{\prime}}^{*}\left(\Omega ; v_{r}\right) f_{j_{1} m \rightarrow j^{\prime} m^{\prime}}\left(\Omega ; v_{r}\right) d \Omega,
$$

in which $\Omega$ represents the scattering solid angle, and $f$ the scattering amplitude. When $m=m_{1}$ and $m^{\prime}=m_{1}^{\prime}$ the $\sigma_{j m m_{1} \rightarrow j^{\prime} m^{\prime} m_{1}^{\prime}}\left(v_{r}\right)$ are collision frame cross- sections. After some algebraic manipulation, Eq. (10) can 
be converted to a more convenient representation in order to treat the polarised arrays of molecules created by single photon absorption,

$$
\begin{aligned}
\underset{j \rightarrow j^{\prime}}{K}\left(v_{r}\right)= & \sum_{m, m^{1}, m^{\prime}, m^{1}, n}(-1)^{\left(j+j^{\prime}-m_{1}-m_{1}^{\prime}\right)}(2 K+1)^{1 / 2}\left(2 K^{\prime}+1\right)^{1 / 2}(2 n+1) \\
& \times\left(\begin{array}{lll}
j & j & K \\
m & m_{1} & M
\end{array}\right)\left(\begin{array}{lll}
j^{\prime} & j^{\prime} & K^{\prime} \\
m^{\prime} & m_{1}^{\prime} & M
\end{array}\right)\left(\begin{array}{lll}
K & K^{\prime} & n \\
0 & 0 & 0
\end{array}\right) \\
& \times\left(\begin{array}{lll}
K & K^{\prime} & n \\
M & -M & 0
\end{array}\right) \sigma_{j m m_{1} \rightarrow j^{\prime} m^{\prime} m_{1}^{\prime}}\left(v_{r}\right)
\end{aligned}
$$

where $K$ and $K^{\prime}$ refer to the state multipole rank before and after collision and $n$ refers to the translational order of the collision environment (see below). Separating out the $\alpha$ dependence of $\sigma_{j \mu \rightarrow j^{\prime} \mu^{\prime}}$ Eq. (9) becomes,

$$
\begin{aligned}
k_{j \rightarrow j^{\prime}}^{K \leftrightarrow K^{\prime}}\left(v_{r}\right)= & \int_{0}^{\infty} \sum_{m, m^{1}, m^{\prime}, m^{1}, n} v_{r}^{3} e^{-\beta v_{r}^{2}}(-1)^{\left(j+j^{\prime}-m_{1}-m^{\prime}\right)} \\
& \times(2 K+1)^{1 / 2}\left(2 K^{\prime}+1\right)^{1 / 2}(2 n+1) \\
& \times\left(\begin{array}{lll}
j & j & K \\
m & m_{1} & M
\end{array}\right)\left(\begin{array}{lll}
j^{\prime} & j^{\prime} & K^{\prime} \\
m^{\prime} & m_{1}^{\prime} & M
\end{array}\right)\left(\begin{array}{lll}
K & K^{\prime} & n \\
0 & 0 & 0
\end{array}\right) \\
& \times\left(\begin{array}{lll}
K & K^{\prime} & n \\
M & -M & 0
\end{array}\right) \sigma_{j m m_{1} \rightarrow j^{\prime} m^{\prime} m_{1}^{\prime}}\left(v_{r}\right) d v_{r} \\
& \times \int_{-1}^{1} e^{\beta v_{2} x^{2}} e^{-M_{a}\left(v_{m z}-v_{r} x^{2}\right)} P_{n}(x) d x .
\end{aligned}
$$

The CF quantities $\sigma_{j m m_{1} \rightarrow j^{\prime} m^{\prime} m_{1}^{\prime}}\left(v_{r}\right)$ may be expressed in terms of $T$ matrix elements:

$$
\begin{aligned}
\sigma_{j m m_{1} \rightarrow j^{\prime} m^{\prime} m_{1}^{\prime}}= & \left.\frac{\pi}{k_{j}^{2}}\right|_{J, J_{1}, l, l_{1}, l^{\prime}, m^{\prime}} i^{l_{1}-l}(2 J+1)\left(2 J_{1}+1\right)(2 l+1)^{1 / 2}(2 l+1)^{1 / 2} \\
& \times\left(\begin{array}{llll}
j & l & J \\
m & 0 & -m
\end{array}\right)\left(\begin{array}{llll}
j & l_{1} & J_{1} \\
m_{1} & 0 & -m_{1}
\end{array}\right)\left(\begin{array}{lll}
j^{\prime} & l^{\prime} & J \\
m^{\prime} & \tilde{m} & -m
\end{array}\right) \\
& \times\left(\begin{array}{lll}
j^{\prime} & l^{\prime} & J_{1} \\
m_{1}^{\prime} & \tilde{m} & -m_{1}
\end{array}\right) T_{j l, j^{\prime} l^{\prime}}^{J} T_{j l_{1}, j^{\prime} l^{\prime}}^{*},
\end{aligned}
$$

where the $T_{j l, j^{\prime} l^{\prime}}^{J}$ are labelled in the total angular momentum, (i.e. orbital + molecular rotation) $J$, representation, and $l$ and $l^{\prime}$ are the orbital angular momenta before and after collision. Taking each $n$ component individually, we find by substitution of Eq. (13) into (11) 
and some angular momentum algebra, that

$$
\begin{aligned}
& \sigma_{j \rightarrow j^{\prime}}^{n K \rightarrow K^{\prime}}\left(v_{r}\right)=\frac{\pi}{k_{j}^{2}}\left(\begin{array}{lll}
K & K^{\prime} & n \\
0 & 0 & 0
\end{array}\right)(2 K+1)^{1 / 2}\left(2 K^{\prime}+1\right)^{1 / 2} \\
& \times \sum_{k, k^{\prime}, q}(2 k+1)^{1 / 2}\left(2 k^{\prime}+1\right)^{1 / 2}-1^{K+k^{\prime}-q}\left(\begin{array}{lll}
k & k^{\prime} & n \\
-q & q & 0
\end{array}\right) \\
& \times\left\{\begin{array}{lll}
j & j & K \\
k & k^{\prime} & n \\
j^{\prime} & j^{\prime} & K^{\prime}
\end{array}\right\} T_{j, j^{\prime}}^{k, q}\left(v_{r}\right) T_{j, j^{\prime}}^{* k^{\prime}, q}\left(v_{r}\right)
\end{aligned}
$$

where

$$
T_{j, j^{\prime}}^{k, q}\left(v_{r}\right)=\sum_{j, l, l^{\prime}} i^{2 j-l}(2 l+1)^{1 / 2}(2 J+1)\left(\begin{array}{lll}
l^{\prime} & l & J \\
q & 0 & -q
\end{array}\right)\left\{\begin{array}{lll}
l & j & J \\
j^{\prime} & l^{\prime} & k
\end{array}\right\} T_{j l, j^{\prime} l^{\prime}}^{J}\left(v_{r}\right) .
$$

This yields from Eq. (12) the final expression for the LF multipolar rate constant

with

$$
\begin{aligned}
& k_{j \rightarrow j^{\prime}}^{n K} K^{\prime}\left(v_{m z}\right)=C \int_{0}^{\infty} v_{r}^{3} e^{-\beta v_{r}^{2}} \sigma_{j \rightarrow j^{\prime}}^{n K} K^{\prime}\left(v_{r}\right) d v_{r} \\
& \times \int_{-1}^{1} e^{\beta v_{r}^{2} x^{2}} e^{-M_{a}\left(v_{z}-v_{r} x\right)^{2}} P_{n}(x) d x,
\end{aligned}
$$

$$
C=\left(\frac{M_{a}}{2 \pi k T}\right)^{3 / 2}\left(\frac{M_{m}}{2 \pi k T}\right)
$$

and where $x=\cos \alpha$.

In the case of a totally isotropic velocity distribution the second integral in Eq. (15) vanishes for $n \geq 1$. If all $n \geq 1$ terms are neglected Eq. (15) reduces to an expression for the rate constant previously derived by Smith et al. ${ }^{7}$ which will not in general be valid unless the cross-sections $\sigma_{j \rightarrow j^{\prime}}^{n K} K^{\prime}$ for $n>0$ are small.

The most important consequence of Eqs. (14) and (15) is the inclusion of the extra index, $n$, as also derived by Alexander ${ }^{8}$ and independently by Monchick ${ }^{9}$ in the description of collision dynamics in non-spherically symmetric collision environments. This index relates to the symmetry order of the collisional interaction in which $n=0$ corresponds to a spatially isotropic relative velocity distribution. The higher order $n$ terms are due to the velocity selection along the laser beam axis, and they occur in other situations for which velocity distributions are anisotropic. ${ }^{10}$ The first 3-j symbol in Eq. (14) indicates the coupling of initial and final state multipoles through the translational order $n$. For $n=0, K=K^{\prime}$, which means that state multipoles of 


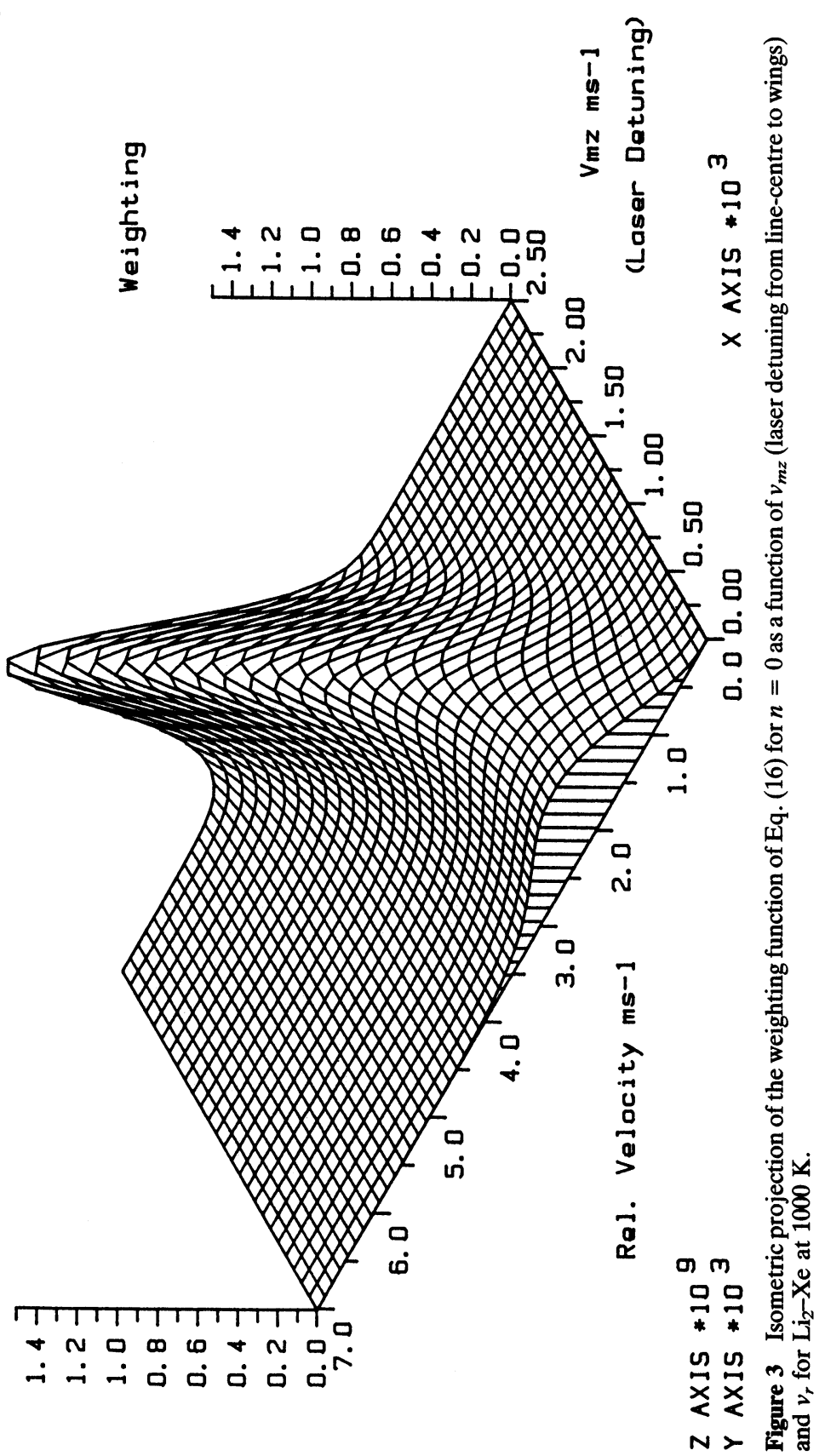


different rank formed from the rotational angular momentum vectors are transferred independently on collision. However, when $n>0$ multipoles of different order can couple. Inspection of Eq. (14) reveals that the sum over $q$ collapses to zero for odd values of $n$ as the $T$-matrix is symmetric $\left(j+l=j^{\prime}+l^{\prime}\right)$. Hence $K+K^{\prime}$ must be even and $n=0,2$, $4,6 \ldots$ The overall parity restrictions for homonuclear diatomic molecules are that odd multipoles can only couple with odd and even with even. Therefore in addition to the standard $\sigma_{j \rightarrow j^{\prime}}^{K}$ multipolar cross-sections, ${ }^{5}$ cross terms such as $\sigma_{j \rightarrow j^{\prime}}^{0 \rightarrow 2}, \underset{j \rightarrow j^{\prime}}{2 \rightarrow 0}$, and $\underset{j \rightarrow j^{\prime}}{1 \rightarrow 1}$, become non-zero in anisotropic collision environments.

The weighting function for each $n$ term, (normalised for each $v_{m z}$ ) as a function of $v_{r}$, is given by:

$$
W\left(v_{r} ; n\right)=v_{r}^{3} e^{-\beta v_{r}^{2}} \int_{-1}^{1} e^{\beta v_{r}^{2}} x^{2} e^{-M_{a}\left(v_{z}-v_{r} x\right)^{2}} P_{n}(x) d x
$$

This is plotted as a function of $v_{m z}$ and $v_{r}$ for the $\mathrm{Li}_{2}-\mathrm{Xe}$ system at $1000 \mathrm{~K}$ for the $n=0$ term in Figure 3 . The mass ratio $14: 131$ for $\mathrm{Li}_{2}: \mathrm{Xe}$ means that when one excites in the wings of the Doppler profile the major contribution to the relative velocity comes from the molecular motion, consequently the relative speed distribution is far from thermal. Figure 4 displays the $n=2$ weighting for the same system and it is of interest to note the negative weighting at line centre. The relative ratio $\frac{n=2}{n=0}$ changes over the Doppler profile from $\simeq-0.4$ to $\simeq 0.8$ and at $\simeq 800 \mathrm{~ms}^{-1}$ is 0 . Similar calculations for $\mathrm{Li}_{2}-\mathrm{He}$ at $1000 \mathrm{~K}$ indicate that $\frac{n=2}{n=0}=0.5$ at large $v_{z}$ and $\frac{n=2}{n=0}=-0.1$ at line centre, despite the unfavourable mass ratio. The higher order $n$ terms have even smaller (and more complex) weighting functions, and may be ignored for the present.

The physical implications of the extra anisotropic components to the multipole cross-sections are now considered. As we have seen, single photon excitation creates a polarised array of molecules described fully by some combination of the first three state multipoles. In a totally isotropic collision environment each state multipole relaxes independently and this is observed as a reduced polarisation in the emission from the excited state. ${ }^{5,11,12}$ In this situation a collision between a structureless atom and a diatomic molecule causes the initial molecular polarisation to be converted into a "polarised" relative velocity distribution.

In a situation where the initial velocity vectors are spatially anisotro- 

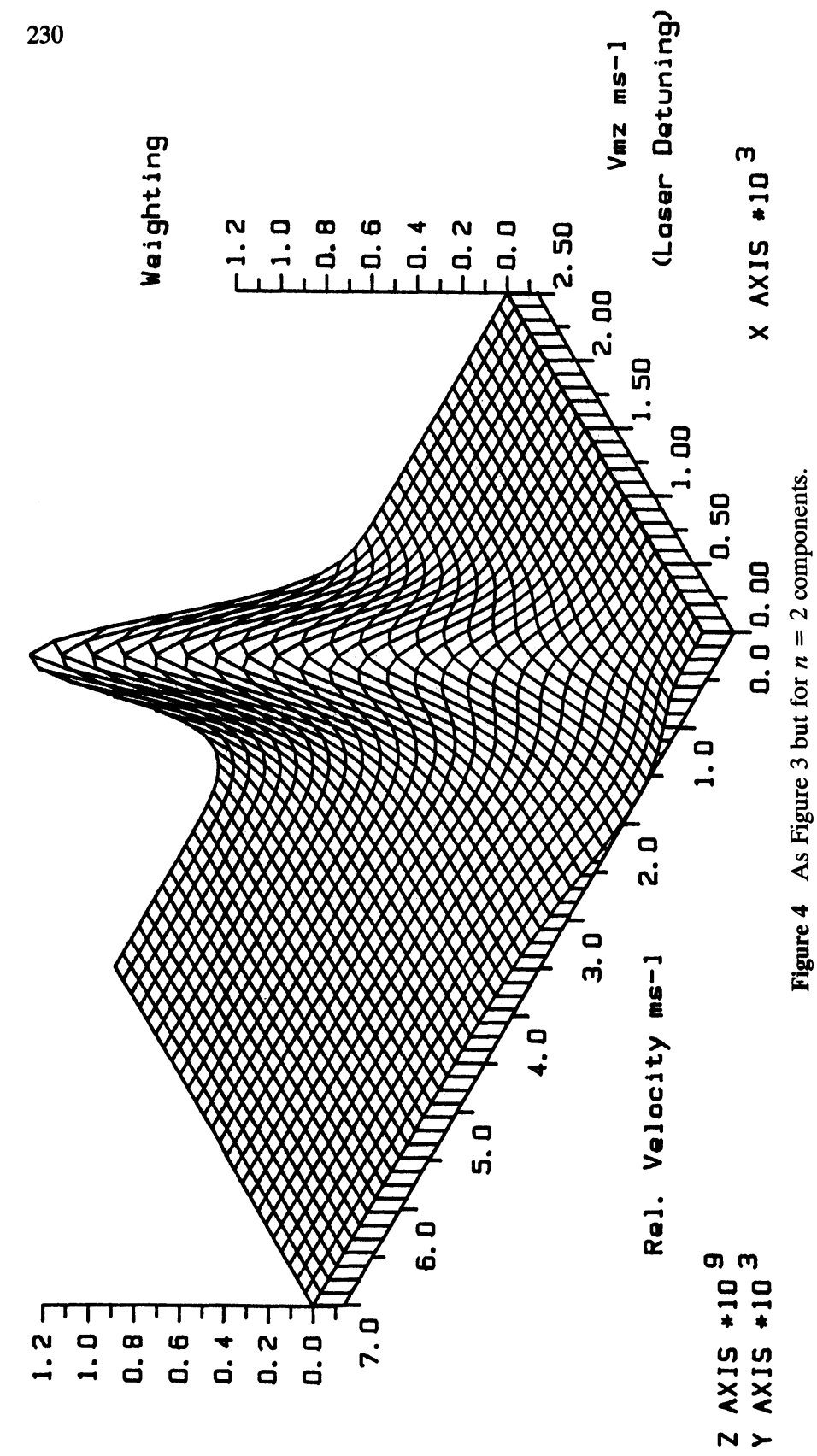
pic it is evident that the reverse process can occur. Here, loss of relative velocity polarisation can cause increased angular momentum polarisation. This theory is embodied in the translational internal coupling scheme of Coombe et al. ${ }^{13}$ where the velocity vectors are coupled to the rotational angular momentum of the molecule. In a system where the $\bar{v}_{r}$ are not randomly distributed there will be two contributing terms to the overall cross-section. Firstly there will be a term which describes how population and polarisation are transferred on collision. This is the term which is applicable to the case of a spherically symmetric velocity distribution $(n=0)$. It would theoretically be possible for the entire $j$ state to be collisionally transferred while the distribution of magnetic sub-levels is totally conserved (i.e. polarisation retained) but the ensemble of molecular angular momentum vectors can never become more polarised by this process. Secondly, the $n \neq 0$ terms describe how the anisotropy of the relative velocity distribution leads to a coupling of state multipoles of different rank so that they may interconvert making it possible for the molecular polarisation to increase.

The above two points hold for any non-isotropic collision process. For beam-gas experiments the possible creation of molecular orientation is expected to be of even greater importance as $\bar{v}_{r}$ is more precisely selected. Alexander ${ }^{6}$ has derived the expressions for RET of a molecular beam impinging on a thermal atomic bath and found, as expected, that there is interconversion between state multipoles of different rank.

The weighting on each $n>0$ component is dependent on the probability of finding the relative velocity at some angle to the laser axis. This may be expressed physically in terms of the correlations between the vectors of relative motion and rotational angular momentum. If the laser is tuned to the centre of the Doppler profile, where $v_{m z}=0$, then the molecular velocity must lie in a plane perpendicular to the direction of laser propagation. The total relative velocity however is a function of both atomic and molecular velocity so that on average it will be centred around the perpendicular plane. If however, the laser frequency is tuned to the wings of the Doppler profile, molecules with large $v_{m z}$ and travelling nearly parallel to the laser direction are selected. In this circumstance the most probable relative velocity vector lies close to the laser propagation direction, the effect being most pronounced if $M_{a} \gg M_{m}$. Any polarisation created in these two extremes is expected to be $90^{\circ}$ out of phase since $\bar{v}_{r}$ has 


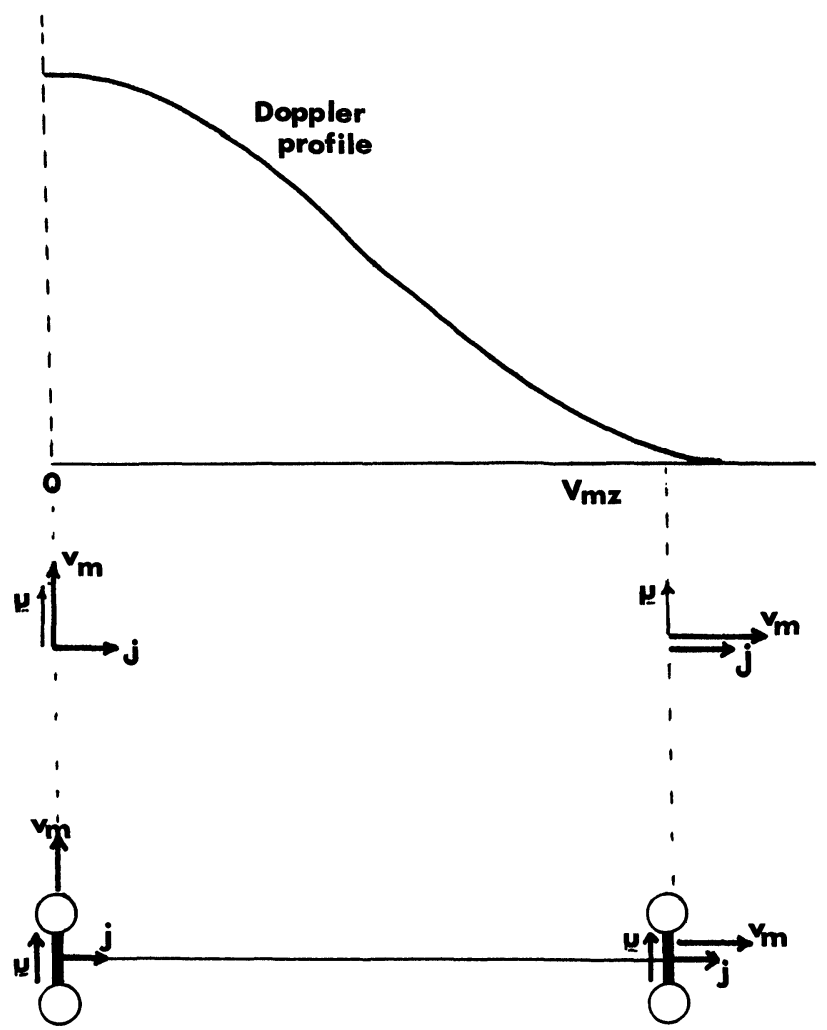

Figure 5 The relative orientations of $\bar{j}$ and $\bar{v}_{m}$ are a function of laser detuning. Illustrated here for a parallel transition, $\mu$ is the transition moment.

changed by $\simeq 90^{\circ}$ with respect to the LF quantisation axis. This may account for the change in sign of the $n=2$ weighting functions of Figure 4. A diatomic molecule having its electronic transition moment parallel to the molecular axis will be aligned perpendicular to the laser propagation direction when excited by a linearly polarised laser. At line centre, $\bar{j}$ and $\bar{v}_{m}$, will be at $90^{\circ}$ whilst in the wings they will be nearly collinear. This is illustrated in Figure 5.

\section{EXPERIMENTAL AND RESULTS}

The molecule chosen for study was $\mathrm{Li}_{2}$, excited into selected rotational states of the $A^{1} \Sigma_{u}^{+}$state, in collision with $\mathrm{Xe}$ atoms. This gives a 
particularly favourable mass ratio in which the relative velocity can be determined almost entirely by the motion of the diatomic molecule. A single-mode tunable dye laser (Coherent model 699-29) was used as the excitation source; the laser linewidth is $<1 \mathrm{MHz}$, which results in a speed selection of about $0.5 \mathrm{~m} \mathrm{~s}^{-1}$ along the laser beam axis. The $\mathrm{Li}_{2}$ was contained in a heated stainless steel cell at about $1000 \mathrm{~K}$. Collisional energy transfer was monitored using a $3 / 4 \mathrm{~m}$ double grating monochromator (Spex model 1402) to resolve fluorecence following $\Delta j=2,4, \ldots$ transitions. The polarisation of the fluorescent light is determined by means of a photo-elastic modulator and analysing polariser placed in the optical train before the monochromator slits. ${ }^{14}$

In outline, the experiment was conducted as follows. The monochromator was set to collect emission from a single rotational level corresponding to a chosen $\Delta j$. The data (intensity and polarisation) were taken as the excitation laser scanned the full Doppler profile of a chosen parent rotational state $(j=6,20$ and 32). The deconvolution routine used to extract the energy-dependence of the RET crosssections requires very "clean" data and a number of techniques were employed to obtain the best quality spectra consistent with the single pass experiment that is needed to preserve polarisation. These and the correction routines employed are more fully described in a forthcoming article. ${ }^{15}$

Expressions such as Eq. (15) for rates of multipolar transfer (ignoring alignment and cross-term effects) may be deconvoluted to yield state-to-state cross-sections as a function of relative velocity. This has been demonstrated by Smith et al. using a Fourier transform technique. ${ }^{1}$ However these authors have applied this solely to the extraction of population $(K=0)$ multipolar constants. Briefly the requirement is that the ratio ${ }^{f} R_{j \rightarrow j^{\prime}}^{K \leftrightarrow}\left(v_{z}\right)$ be exactly replicated by an even order polynomial expansion:

$$
f_{R_{j \rightarrow j^{\prime}}^{K}}^{K K_{z}}\left(v_{z}\right)=\sum_{m=0}^{N} a_{m} v_{z}^{2 m} .
$$

Following the Fourier transform the cross-sections can then be calculated by:

$$
\begin{aligned}
\underset{j \rightarrow j^{\prime}}{K}\left(v_{r}\right)= & \sum_{p=0}^{N} \frac{v_{r}^{2 p-1}}{(2 p+1) !} \sum_{n=p}^{N} \\
& \times \frac{a_{n}(2 n) !(-r / 2)^{n-p}[(2 m+1) r+2(m-p)]}{r(m-p) !},
\end{aligned}
$$


with

$$
r=\frac{M_{\mathrm{Li}_{2}}}{M_{\mathrm{Xe}}}
$$

Smith et al. ${ }^{7}$ have already described the limitations and resolution of the technique so only the major points are outlined below. Firstly, the range of $v_{r}$ is strictly limited by the range of $v_{m z}$, which for this experiment was $\simeq 500 \mathrm{~ms}^{-1}$ to $\simeq 2300 \mathrm{~ms}^{-1}$. Secondly, the resolution is determined by the order of the polynomial fit. Ideally the order of the polynomial should be $m \geq 12$. However, for cross-sections with simple functional forms $m \geq 7$ is sufficient. Thirdly, noise is the largest source of error as this will lower the order of the polynomial available for the fit. A very high order expansion will inevitably lead to unrealistic oscillations in the deconvoluted cross-sections due to the polynomial "fitting the noise".

When fitting the ratios a certain trade off had to be made with noise and the highest order polynomial. The ratios were fitted with successively higher order polynomials until a convergence was reached in the deconvoluted cross-sections. This was always the largest order polynomial before the experimental noise forced the cross-sections to oscillate wildly. Typically a polynomial of order $m=9$ was employed. The largest value of $\Delta j$ was determined by the fluorescence intensity of the satellite feature.

Therefore in summary the experiment and data handling were similar in nature to the VSDS method of Smith et al. ${ }^{1}$ except that circularly polarised excitation was used and explicitly detected, in this way cross-sections for tranfer of orientation could be measured and deconvoluted separately.

Representative raw data traces are shown in Figure 6. The three traces show the population signal (total intensity), orientation (circular polarisation), and the ratio of the two. The data shown are for the transition $\Delta j=6$ from $j_{\text {initial }}=6$. This case demonstrates some of the most pronounced effects observed in all of the transitions studied. The difference in lineshape between the orientation and population signals is very marked and this is strongly accentuated in the ratio plot. Normalised deconvoluted population and orientation cross-sections for this transition are shown in Figure 7.

Both cross-sections can be seen to exhibit a threshold, rise sharply to a maximum and then fall off more slowly with collision energy. The 


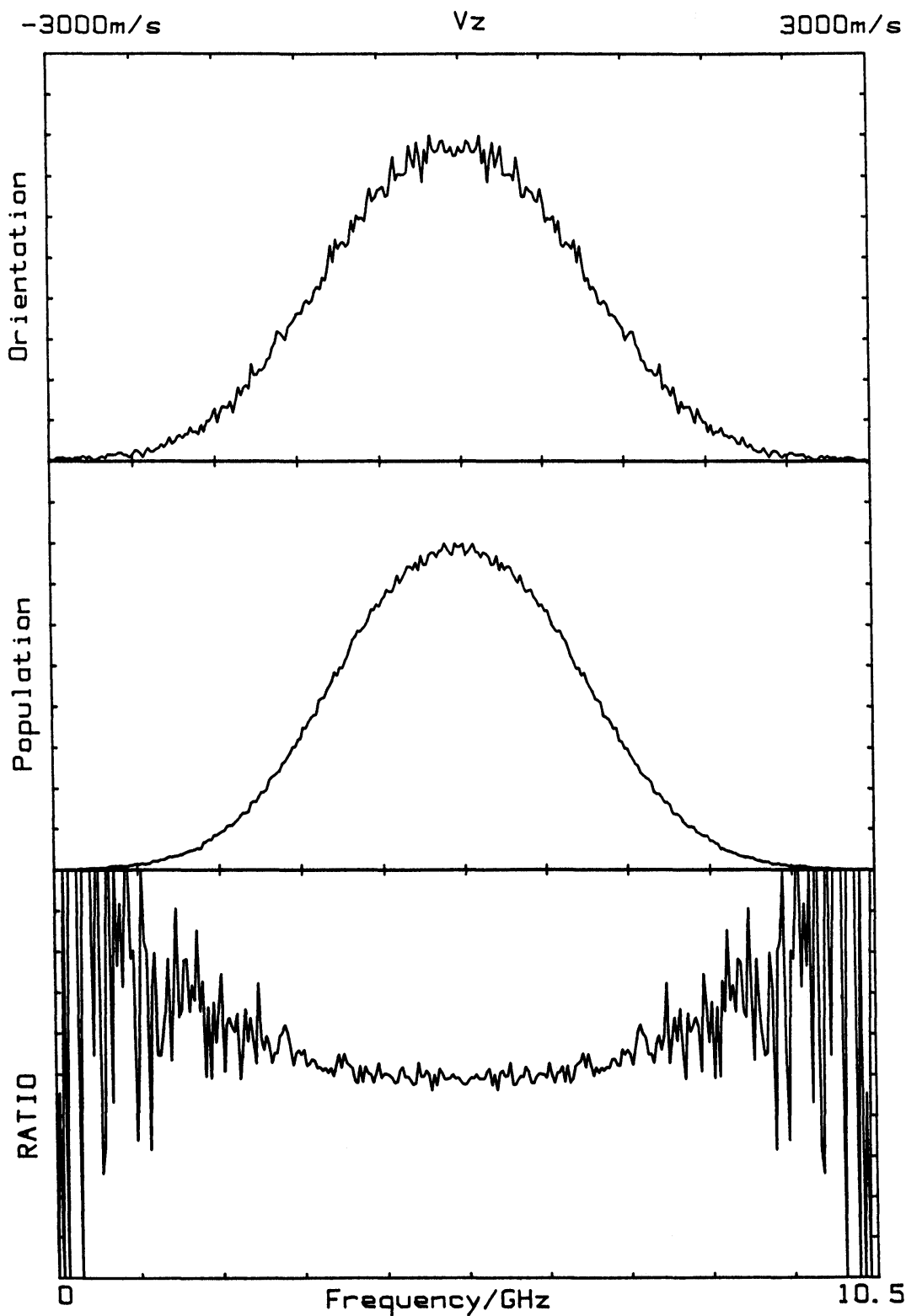

Figure 6 Circularly polarised emission and total fluorescence intensity of the $\Delta j=6$ satellite following excitation of $j=6$ in the $A^{1} \Sigma_{u}^{+} v=18$ state of $\mathrm{Li}_{2}$. The third trace shows the ratio of circularly polarised light to total intensity. 


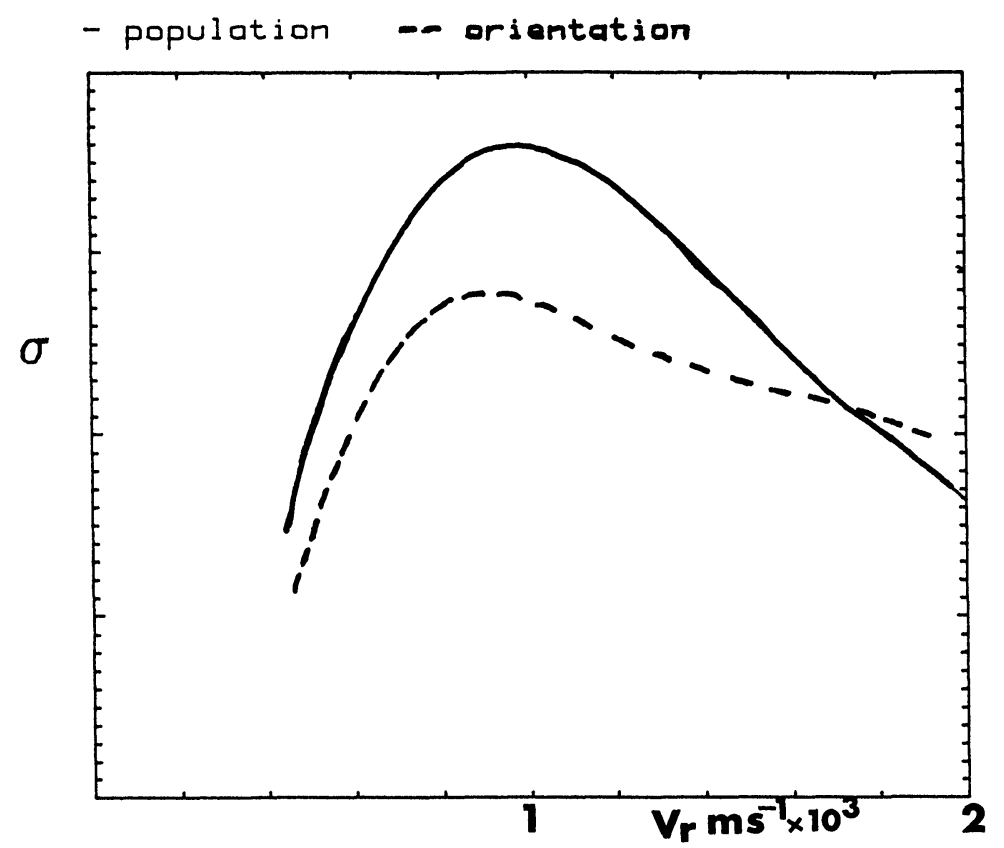

Figure 7 Population and orientation cross-sections for $j=6 \rightarrow 12$ as a function of relative velocity as obtained from the data presented in Figure 6.

threshold is characteristic of endoergic RET processes whilst the fall-off at high energy can be explained by the fact that higher $\Delta j$ transitions become energetically accessible. It also seems likely that the anisotropy of the potential becomes less pronounced at high energy rendering RET less efficient. Since there is known to be a large well depth in the potential for $\mathrm{Li}_{2}{ }^{*}-\mathrm{XE}$ it would seem that the attractive part of the potential may have a strong influence.

It is also evident that the orientation cross-section falls off more slowly with energy than the population cross-section. This indicates that higher energy collisions tend to conserve the LF projection of the rotational angular momentum but it is important to remember that in the velocity selection technique used here high and low energy collisions are associated with different relationships between $j$ and $v_{r}$. The influence of this effect is currently under investigation.

Close coupled calculations have been performed on model systems ${ }^{16}$ and reproduce the energy dependence of population, orientation and 
alignment cross sections. It has also been shown ${ }^{17}$ that for sudden collisions it is possible to scale these cross-sections.

\section{ENTRANCE-EXIT CHANNEL VELOCITY SELECTED EXPERIMENTS}

The techniques outlined above may be extended to provide information on the way in which the direction of the relative velocity vector changes during a collision. In principle, all that is required is the use of a very high resolution detector in the experimental set-up described so that the velocity spread of molecules following collision is reflected by the frequency of the emitted fluorescence. In practice, this is achieved by employing a second laser (co- or counter-propagating to the first) that will absorb from the first excited state at a frequency depending on the molecular velocity component along this laser axis following collision. It has been shown by Gottscho et al. ${ }^{18}$ that the change in molecular velocity component is directly related to the centre of mass scattering angle, $\theta_{c}$ :

$$
\Delta v_{m z}=\left(M_{a} / M\right)\left(v_{r i} \cos \alpha \cos \theta_{c}-v_{r f} \cos \alpha\right),
$$

where

$$
M=M_{a}+M_{m},
$$

as can be seen by referring to Figure 8 . If the energy change is small compared with the collision energy, as is often the case for rotationally inelastic collisions, we have:

$$
\Delta v_{m z}=\left(M_{a} / M\right) v_{r i} \cos \alpha\left(\cos \theta_{c}-1\right)
$$

An experiment along these lines was performed in 1980 by Gottscho et al. ${ }^{18}$ to study $\mathrm{BaO}+\mathrm{Ar} / \mathrm{CO}_{2}$ collisions, and they were able to obtain energy-averaged most-probable angles. In principle, using a more suitable system (e.g. $\mathrm{Li}_{2} / \mathrm{Xe}$ ), it should be possible to extract more detailed information by making use of the velocity selection treatment. We know from Eq. (4) that the probability of finding a speed $v_{r}$ at an angle $\alpha$ to the laser axis, for a given first laser excitation frequency that selects $v_{m z}$, is given by:

$$
\begin{aligned}
W\left(v_{r}, \cos \alpha, v_{m z}\right)= & v_{r}^{2} \exp \left(-\beta v_{r}^{2} \sin ^{2} \alpha\right) \\
& \times \exp \left[-M_{a}\left(v_{m z}-v_{r} \cos \alpha\right)^{2}\right] .
\end{aligned}
$$




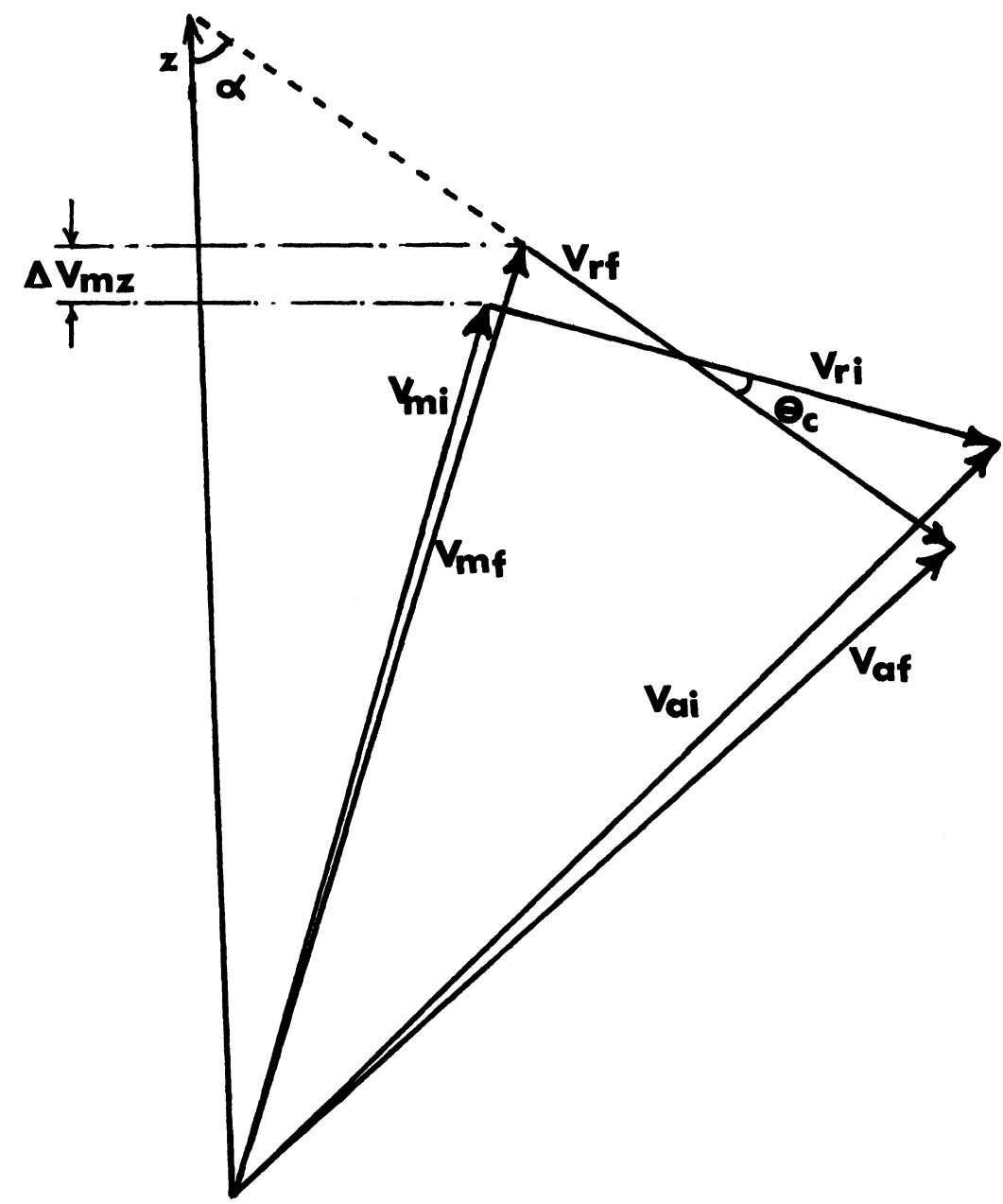

Figure 8 The relationship between the change in the $z$ component of molecular velocity $\Delta v_{m z}$ and the centre of mass frame scattering angle $\theta_{c}$.

Then the probability of selecting an initial molecular velocity component is given by a Lorentzian distribution the parameters of which depend on the dominant homogeneous broadening mechanism. The only probability distribution left to find in order to determine the final lineshape for total emission as a function of the second laser excitation frequency is a quantity proportional to the differential cross-section which we denote by $W\left(\theta_{c}\right)$ and which will be energy dependent. 
Thus far we have calculated lineshapes for model differential crosssections based on the observations of ${ }^{19}$ and calculations by Serri et al. ${ }^{20}$ and have found them highly sensitive to the energy dependence of the differential cross-section. It would seem that the experimental data may be deconvoluted to give energy dependent angular information and furthermore that we may relate polarisation information to the scattering angle information.

\section{CONCLUSIONS}

It has been shown that it is possible to obtain velocity dependent collision cross-sections from thermal systems by using narrow linewidth lasers through the technique of velocity selection by Doppler shift. The method uses the narrow frequency output from a single (longitudinal) mode dye laser to excite different velocity components under the Doppler profile of a transition to a given quantum state whilst monitoring the fluorescence from nearby levels populated by inelastic energy transfer. In this way one of the translational degrees of freedom of the molecule under study can be tightly controlled by the experimenter allowing the extraction of energy dependent crosssections for the energy transfer process.

Early workers who applied the technique in the field of rotational energy transfer were concerned simply with population (i.e. $j-j^{\prime}$ ) cross-sections and did not take into account effects due to the polarisation of the exciting light field. Here we have developed expressions which take polarisation into account and some important consequences follow. Firstly, we have shown that the translational order created in the excited state affects the evolution of state multipole moments and that they can be mixed by collisions rather than evolving independently as in an isotropic collision environment. Secondly, we have demonstrated that the velocity dependence of the multipolar cross-sections may be extracted experimentally, and we have described results for the RET in $\operatorname{Li}_{2}\left(A^{1} \Sigma_{u}^{+}\right)-\mathrm{Xe}$. Our observations indicate that additional dynamical information can be extracted from such polarisation resolved experiments.

Finally we have described a further experiment in which the velocity dependence of the exit channel is probed for a given selected entrance channel velocity. From this it is possible to extract energy dependent 
differential cross-sections for RET of molecules in a thermal cell. Although the angular resolution achievable by this method is nowhere near as good as that possible in crossed molecular beam experiments the collision energy range that may be studied is much larger and the method is of wide applicability.

\section{Acknowledgements}

We are grateful to the Science and Engineering Research Council for financial support, and for the award of three Research Studentships (CPF, AT, KLR) and an Advanced Research Fellowship (BJW).

\section{References}

1. N. Smith, T. P. Scott and D. E. Pritchard, J. Chem. Phys. 81, 1229 (1984).

2. P. L. Houston, J. Phys. Chem. 91, 5388 (1987).

3. J. C. Light, J. Ross and K. E. Shuler, "Kinetic processes in gases and plasmas," ed. A. R. Hochstim, Academic Press, 1969.

4. U. Fano and G. Racah, "Irreducible tensorial sets," Academic Press, 1959.

5. M. D. Rowe and A. J. McCaffery, Chem. Phys. 43, 35 (1979).

6. M. H. Alexander, P. J. Dagdigian and A. E. DePristo, J. Chem. Phys. 66, 59 (1977).

7. N. Smith, T. A. Brunner, R. D. Driver and D. E. Pritchard, J. Chem. Phys. 69, 498 (1978).

8. M. H. Alexander and S. L. Davis, J. Chem. Phys. 78, 6768 (1983).

9. L. Monchick, J. Chem. Phys. 75, 3377 (1981).

10. A. J. Bain, PhD Thesis, Sussex, 1984.

11. S. J. Silvers, R. A. Gottscho and R. W. Field, J. Chem. Phys. 74, 6000 (1981).

12. A. J. McCaffery, M. J. Proctor, E. A. Seddon and A. Ticktin, Chem. Phys. Lett. 132, 185 (1986).

13. D. A. Coombe, R. F. Snider and D. C. Sanctuary, J. Chem. Phys. 63, 3015 (1975).

14. S. R. Jeyes, A. J. McCaffery and M. D. Rowe, Mol. Phys. 36, 845 (1978).

15. C. P. Fell, A. J. McCaffery, A. Ticktin and B. J. Whitaker, J. Chem. Phys. (to be submitted).

16. A. Ticktin, B. J. Whitaker and A. J. McCaffery, Chem. Phys. Lett. 139, 571 (1987).

16. J. Derouard and N. Sedeghi, Chem. Phys. 88, 171 (1984).

18. R. A. Gottscho, R. W. Field, R. Bacis and S. J. Silvers, J. Chem. Phys. 73, 599 (1980).

19. J. A. Serri, C. H. Becker, M. B. Elbel, J. L. Kinsey, W. P. Moskowitz and D. E. Pritchard, J. Chem. Phys. 74, 5116 (1981).

20. J. A. Serri, R. M. Bilotta and D. E. Pritchard, J. Chem. Phys. 77, 2940 (1982). 